\title{
ON INVARIANT MEASURES OF NONLINEAR MARKOV PROCESSES ${ }^{1}$
}

\author{
N.U. AHMED and XINHONG DING \\ University of Ottawa \\ Department of Electrical Engineering \\ and Department of Mathematics \\ Ottawa, Ontario K1N 6N5, CANADA
}

\begin{abstract}
We consider a nonlinear (in the sense of McKean) Markov process described by a stochastic differential equations in $R^{d}$. We prove the existence and uniqueness of invariant measures of such process.
\end{abstract}

Key words: Stochastic differential equations, McKean-Vlasov equation, invariant measures.

AMS (MOS) subject classifications: 60J05, 60J25, 60J60, $60 \mathrm{H} 10,28 \mathrm{C} 10$.

\section{INTRODUCTION}

In this paper we study the asymptotic property of a nonlinear Markov process described by the following stochastic differential equation in $d$ dimensional Euclidean space $R^{d}$ :

$$
\left\{\begin{array}{l}
d X(t)=[-A X(t)+f(X(t), \mu(t))] d t+d W(t), \quad t \geq 0 \\
\mu(t)=\text { probability law of } X(t)
\end{array}\right.
$$

where $W$ is a standard $d$-dimensional Wiener process; $A$ is a $d \times d$-dimensional matrix; $f$ is an appropriate $R^{d}$-valued function defined on $R^{d} \times M_{2}\left(R^{d}\right)$. Here $M_{2}\left(R^{d}\right)$ denotes the space of all probability measures on $R^{d}$ which have finite second moments. Under mild conditions, the above equation has a unique solution $X=\{X(t), t \geq 0\}$. We are interested in the stationary behavior of its probability distribution $\mu(t)$, as a measure-valued function. In particular, we want to find the conditions that ensure the existence and uniqueness of invariant measures for the stochastic differential equation (1).

\footnotetext{
${ }^{1}$ Received: October, 1993. Revised: December, 1993.
} 
By far, there are many papers in the literature which were devoted to the studies of invariant measures of Markov processes, both in finite and infinite dimensional spaces. Some of them are listed in the references ([3], [10], [15], [17], [19], [20], [21], [24], [26]). There are also several research papers and one monograph which were devoted to the study of long time behavior of nonlinear stochastic differential equations of McKean type ([7], [22], [23]). But the drift terms in these models are usually assumed to be of gradient type, so the associated invariant measures can be written down explicitly. To the knowledge of the authors, for nongradient type drift such as the one in (1), the problems related to the invariant measures have not been studied in the literature.

It is important to point out at the outset that may of the standard techniques and results on invariant measures for Markov processes cannot be applied to model (1) directly without appropriate modifications because (1) is not a Markov process in the usual sense. We also want to point out that this model provides a first step towards a better understanding of the behavior of similar stochastic evolution equation in a Hilbert space where $-A$ is the infinitesimal generator of $C_{0}$-semigroup. This infinite dimensional model is currently under investigation.

Our main results (see theorem 3 in section 4 ) give sufficient condition for existence and uniqueness of invariant measures of the system (1).

The proof of the existence theorem is based on a general criterion (see theorem 2 of section 3) on the existence of invariant measures for McKean type of nonlinear Markov processes, which is of independent interest. An example is given in section 4 to indicate that the conditions obtained in this paper are only sufficient conditions.

The rest of this paper is organized as follows. In section 2 we prove the existence and uniqueness of solutions of the stochastic differential equation (1). In section 3 we prove a general theorem which ensures the existence and uniqueness of invariant measures for McKean-Vlasov nonlinear stochastic differential equations. In section 4 we apply this theorem to the nonlinear Markov process determined by equation (1) and give a simple example. 


\section{STOCHASTIC DIFFERENTIAL EQUATION}

We first introduce some notations. Throughout this paper $R^{d}$ always denotes $d$-dimensional real Euclidean space with scalar product $\langle\cdot, \cdot\rangle$ and norm 1.1. $\mathscr{B}\left(R^{d}\right)$ denotes the Borel sigma-algebra of subsets of $R^{d} . R^{d \otimes d}$ denotes the space of $d \times d$ real matrices. We denote by $C^{\infty}\left(R^{d}\right), C_{b}\left(R^{d}\right), C_{K}^{\infty}\left(R^{d}\right)$ the space of real valued functions on $R^{d}$ which are smooth, bounded continuous, and smooth functions with compact supports, respectively.

Let $(\Omega, F, P)$ be a complete probability space equipped with the filtration $\left\{\mathcal{F}_{t}: t \geq 0\right\}$ of nondecreasing sub-sigma algebras of $\mathcal{F}$. The expectation with respect to $P$ will be denoted by $E$. Let $W=\{W(t): t \geq 0\}$ be a standard $d$ dimensional Wiener process defined on this probability space such that $W$ is adapted to $\left\{\digamma_{t}: t \geq 0\right\}$.

Let $M\left(R^{d}\right)$ denote the space of all probability measures on $R^{d}$ furnished with the usual topology of weak convergence. Let $M_{2}\left(R^{d}\right)$ be the collection of all $\mu \in M\left(R^{d}\right)$ satisfying

$$
\|\mu\|_{2}=\left\{\int_{R^{d}}|x|^{2} \mu(d x)\right\}^{\frac{1}{2}}<+\infty .
$$

The space $M_{2}\left(R^{d}\right)$ is equipped with a topology determined by a special metric $\rho_{2}(P, Q)$ defined by

$$
\rho_{2}(P, Q)=\inf \left\{\int_{R^{d} \times R^{d}}\left(|x-y|^{2} \wedge 1\right) F(d x \times d y)\right\}^{\frac{1}{2}},
$$

where $P, Q \in M_{2}\left(R^{d}\right)$ and the infimum is taken over the space $M\left(R^{d} \times R^{d}\right)$ of all probability measures $F$ on $R^{d} \times R^{d}$ such that $F$ has marginal measures $P$ and $Q$. It is known [9] that $\left(M_{2}\left(R^{d}\right), \rho_{2}\right)$ is a complete metric space and a sequence $\mu_{n}$ of probability measures converges in $\left(M_{2}\left(R^{d}\right), \rho_{2}\right)$ to a probability measure $\mu$ if and only if $(a) \mu_{n}$ converges to $\mu$ weakly in $M\left(R^{d}\right)$ and $(b)$ the second moments $\left\|\mu_{n}\right\|_{2}^{2}$ converges to $\|\mu\|_{2}^{2}$.

We denote by $C\left([0, \infty), M_{2}\left(R^{d}\right)\right)$ the metric space of continuous functions from $[0, \infty)$ to $M_{2}\left(R^{d}\right)$ with the metric:

$$
D(\mu(\cdot), \nu(\cdot))=\sum_{N=1}^{\infty} \frac{1}{2^{N}}\left(\sup _{0 \leq t \leq N} \rho_{2}(\mu(t), \nu(t)) \wedge 1\right)
$$




$$
=\sum_{N=1}^{\infty} \frac{1}{2^{N}}\left(D_{N}((\mu(t), \nu(t)) \wedge 1)\right.
$$

where $\mu(\cdot)$ and $\nu(\cdot)$ belong to $C\left([0, \infty), M_{p}\left(R^{d}\right)\right)$.

We consider the Itô stochastic differential equation (1) and assume that

(A1): The operator $A$ is a $d \times d$-dimensional matrix such that the associated semigroup $S(t)=\exp (-A t), t \geq 0$, of bounded linear operators on $R^{d}$ satisfies

$$
\|S(t)\| \leq \exp (-\omega t)
$$

for some strictly positive constant $\omega$, where $\|S(t)\|$ denotes the operator norm.

$(A 2)$ : The function $f: R^{d} \times M_{2}\left(R^{d}\right) \rightarrow R^{d}$ satisfies

$$
\begin{gathered}
|f(x, \mu)-f(y, \nu)|^{2} \leq k\left(|x-y|^{2}+\rho_{2}^{2}(\mu, \nu)\right) \\
|f(x, \mu)|^{2} \leq l\left(1+|x|^{2}+\|\mu\|_{2}^{2}\right)
\end{gathered}
$$

where $k$ and $l$ are positive constants.

Theorem 1: $\quad$ Suppose that conditions $(A 1)$ and $(A 2)$ are satisfied. Then for any $x \in L_{2}\left(\Omega, \mathcal{F}_{0}, P ; R^{d}\right)$, stochastic differential equation (1) has a unique solution $X=\{X(t): t \geq 0\}$ with $X(0)=x$.

$$
\begin{aligned}
& \text { Proof: We use the classical Picard iteration scheme. Define } \\
& \left\{\begin{array}{l}
X_{0}(t)=S(t) x \\
X_{n}(t)=S(t) x+\int_{0}^{t} S(t-s) f\left(X_{n-1}(s), \mu_{n-1}(s)\right) d s+\int_{0}^{t} S(t-s) d W(s) \\
n=1,2, \ldots
\end{array}\right.
\end{aligned}
$$

where $\mu_{n}(t)$ denotes the distribution of $X_{n}(t)$. Then

$$
X_{n+1}(t)-X_{n}(t)=\int_{0}^{t} S(t-s)\left[f\left(X_{n}(s), \mu_{n}(s)\right)-f\left(X_{n-1}(s), \mu_{n-1}(s)\right)\right] d s
$$

and it follows from the assumptions $(A 1)$ and $(A 2)$ that for any $T>0$,

$$
\begin{gathered}
E\left(\sup _{0 \leq t \leq T}\left|X_{n+1}(t)-X_{n}(t)\right|^{2}\right) \\
\leq T E\left(\int_{0}^{T}\|S(t-s)\|^{2}\left|f\left(X_{n}(s), \mu_{n}(s)\right)-f\left(X_{n-1}(s), \mu_{n-1}(s)\right)\right|^{2} d s\right)
\end{gathered}
$$




$$
\leq T k \int_{0}^{T}\left(E\left|X_{n}(s)-X_{n-1}(s)\right|^{2}+\rho_{2}^{2}\left(\mu_{n}(s), \mu_{n-1}(s)\right)\right) d s .
$$

Since by the definition (3) of metric $\rho_{2}$ we have that

$$
\rho_{2}^{2}\left(\mu_{n}(s), \mu_{n-1}(s)\right) \leq E\left|X_{n}(s)-X_{n-1}(s)\right|^{2},
$$

it is easy to verify that

$$
E\left(\sup _{0 \leq t \leq T}\left|X_{n+1}(t)-X_{n}(t)\right|^{2}\right) \leq 2 T k \int_{0}^{T} E\left(\sup _{0 \leq \theta \leq s}\left|X_{n+1}(\theta)-X_{n}(\theta)\right|^{2}\right) d s .
$$

Writing $\Phi_{n}(t)=E\left(\sup _{0 \leq s \leq t}\left|X_{n}(s)-X_{n-1}(s)\right|^{2}\right)$ and $\alpha=2 T k M^{2}$, we have

$$
\Phi_{n+1}(T) \leq \alpha \int_{0}^{T} \Phi_{n}(t) d t
$$

Hence by repeated substitution of (10) into its definition, we obtain

$$
\Phi_{n+1}(T) \leq \alpha^{n} \frac{T^{n}}{n !} \Phi_{1}(T)
$$

Since

$$
\begin{gathered}
\Phi_{1}(T)=E\left(\sup _{0 \leq t \leq T}\left|X_{1}(t)-X_{0}(t)\right|^{2}\right) \\
\leq 2 T E \int_{0}^{T}\|S(t-s)\|^{2}\left|f\left(X_{0}(s), \mu_{0}(s)\right)\right|^{2} d s+2 \int_{0}^{T}\|S(t-s)\|^{2} d s \\
\leq 2 T l \int_{0}^{T}\left(1+E\left|X_{0}(s)\right|^{2}+\left\|\mu_{0}(s)\right\|{ }_{2}^{2}\right) d s+2 T \\
\leq 2 T l \int_{0}^{T}\left(1+2 E|x|^{2}\right) d s+2 T \\
\leq 2 T l T\left(1+2 E|x|^{2}\right)+2 T \\
\leq C_{T}=\left(a T+b T^{2}\right), \quad a, b>0
\end{gathered}
$$

we have

$$
E\left(\sup _{0 \leq t \leq T}\left|X_{n+1}(t)-X_{n}(t)\right|^{2}\right)=\phi_{n+1}(T) \leq C_{T} \alpha^{n} \frac{T^{n}}{n !}
$$

Thus

$$
P\left\{\sup _{0 \leq t \leq T}\left|X_{n+1}(t)-X_{n}(t)\right|>\frac{1}{2^{n}}\right\} \leq C_{T}(4 \alpha)^{n} \frac{T^{n}}{n !}
$$

By Borel-Cantelli's lemma, the processes $X_{n}(t)$ converge uniformly on $[0, T]$ for arbitrary $T>0$. The limit process $X(t)$ is then continuous and solves equation 
(1). This proves the existence of a solution of equation (1).

To prove uniqueness, we let $X_{1}(t)$ and $X_{2}(t)$ be two solutions of equation (1) such that $X_{1}(0)=X_{2}(0)$. The corresponding distribution of $X_{1}(t)$ and $X_{2}(t)$ will be denoted by $\mu_{1}(t)$ and $\mu_{2}(t)$, respectively. Let $\sigma_{1}^{N}=\inf \left\{t:\left|X_{1}(t)\right| \geq N\right\}$ and $\sigma_{2}^{N}=\inf \left\{t:\left|X_{2}(t)\right| \geq N\right\}$. We show that for each $N=1,2, \ldots, \sigma_{1}^{N}=\sigma_{2}^{N}$ and $X_{1}(t)=X_{2}(t)$ for all $t \leq \sigma_{1}^{N}$. We have

$$
\begin{gathered}
X_{1}\left(t \wedge \sigma_{1}^{N} \wedge \sigma_{2}^{N}\right)-X_{2}\left(t \wedge \sigma_{1}^{N} \wedge \sigma_{2}^{N}\right) \\
=\int_{0}^{t \wedge \sigma_{1}^{N} \wedge \sigma_{2}^{N}} S(t-s)\left[f\left(X_{1}(s), \mu_{1}(s)\right)-f\left(X_{2}(s), \mu_{2}(s)\right)\right] d s
\end{gathered}
$$

so, for any $t \in[0, T]$,

$$
\begin{gathered}
E\left|X_{1}\left(t \wedge \sigma_{1}^{N} \wedge \sigma_{2}^{N}\right)-X_{2}\left(t \wedge \sigma_{1}^{N} \wedge \sigma_{2}^{N}\right)\right|^{2} \\
\left.\leq T k E \int_{0}^{t \wedge \sigma_{1}^{N} \wedge \sigma_{2}^{N}}\left(\left|X_{1}(s)-X_{2}(s)\right|^{2}+\rho_{2}\left(\mu_{1}(s)\right), \mu_{2}(s)\right)\right) d s \\
\leq T k E \int_{0}^{t}\left(\left|X_{1}\left(s \wedge \sigma_{1}^{N} \wedge \sigma_{2}^{N}\right)-X_{2}\left(s \wedge \sigma_{1}^{N} \wedge \sigma_{2}^{N}\right)\right|^{2}\right. \\
\left.\left.+\rho_{2}^{2}\left(\mu_{1}\left(s \wedge \sigma_{1}^{N} \wedge \sigma_{2}^{N}\right)\right), \mu_{2}\left(s \wedge \sigma_{1}^{N} \wedge \sigma_{2}^{N}\right)\right)\right) d s \\
\left.\left.\leq 2 T k \int_{0}^{t} E\left|X_{1}\left(s \wedge \sigma_{1}^{N} \wedge \sigma_{2}^{N}\right)-X_{2}\left(s \wedge \sigma_{1}^{N} \wedge \sigma_{2}^{N}\right)\right|^{2}\right)\right) d s .
\end{gathered}
$$

Hence the Gronwall's inequality yields

$$
E\left|X_{1}\left(t \wedge \sigma_{1}^{N} \wedge \sigma_{2}^{N}\right)-X_{2}\left(t \wedge \sigma_{1}^{N} \wedge \sigma_{2}^{N}\right)\right|^{2}=0, \quad \forall t \in[0, T]
$$

Letting $T \rightarrow \infty$ we obtain

$$
X_{1}\left(t \wedge \sigma_{1}^{N} \wedge \sigma_{2}^{N}\right)=X_{2}\left(t \wedge \sigma_{1}^{N} \wedge \sigma_{2}^{N}\right) \text { a.s. for all } t \geq 0 .
$$

Since $X_{1}$ and $X_{2}$ are continuous processes, we can conclude that $X_{1}(t)=X_{2}(t)$ for all $t \in\left[0, \sigma_{1}^{N} \wedge \sigma_{2}^{N}\right)$. This implies $\sigma_{1}^{N}=\sigma_{2}^{N}$ a.s. and the uniqueness is proved. 


\section{A GENERAL CRITERION FOR INVARIANT MEASURES}

In this section we provide a general result on the existence of invariant measures for the nonlinear Markov processes described by the following Itô equation in $R^{d}$ :

$$
\left\{\begin{array}{l}
d X(t)=b(X(t), \mu(t)) d t+\sigma(X(t), \mu(t)) d W(t), \quad t \geq 0 \\
\mu(t)=\text { probability law of } X(t), \\
\mu_{0}=\text { the initial law of } X(0), \quad \mu_{0} \in M_{2}\left(R^{d}\right),
\end{array}\right.
$$

where $W$ is a $d$-dimensional Wiener process, $b$ and $\sigma$ are continuous functions from $R^{d} \times M_{2}\left(R^{d}\right)$ to $R^{d}$ and $R^{d \otimes d}$, respectively, satisfying the conditions

$$
\begin{gathered}
|b(x, \mu)-b(y, \nu)|^{2}+|\sigma(x, \mu)-\sigma(y, \nu)|^{2} \leq k\left(|x-y|^{2}+\rho_{2}^{2}(\mu, \nu)\right) \\
|b(x, \mu)|^{2}+|\sigma(x, \mu)|^{2} \leq l\left(1+|x|^{2}+\|\mu\|_{2}^{2}\right)
\end{gathered}
$$

where $x, y \in R^{d}, \mu, \nu \in M_{2}\left(R^{d}\right)$ and $k, l$ are two positive constants. Using Picard iterative technique similar to that used in the previous section, one can show that equation (19) has a unique continuous solution.

Let $X$ denote the unique solution of equation (19) and let $\mu(t)$ denote the probability law of $X(t)$. Then by Itô's formula the measure valued function $\mu(\cdot)$ satisfies the McKean-Vlasov equation

$$
\left\{\begin{array}{l}
\frac{d}{d t}\langle\mu(t), \varphi\rangle=\langle\mu(t), L(\mu(t)), \varphi\rangle, \quad t>0, \forall \varphi \in C_{K}^{\infty}\left(R^{d}\right) \\
\mu(0)=\mu_{0}
\end{array}\right.
$$

where for each $\mu \in M_{2}\left(R^{d}\right), L(\mu)$ is given by

$$
L(\mu) \varphi(x)=\frac{1}{2} \sum_{i, j=1}^{d} a_{i j}(x, \mu) \frac{\partial^{2} \varphi(x)}{\partial x_{i} \partial x_{j}}+\sum_{i=1}^{d} b_{i}(x, \mu) \frac{\partial \varphi(x)}{\partial x_{i}}
$$

for $a_{i j}(x, \mu)=\sum_{k=1}^{d} \sigma_{i k}(x, \mu) \sigma_{j k}(x, \mu)$.

Definition 1: A probability measure $\rho \in M\left(R^{d}\right)$ is said to be an invariant measure associated with system (19) if $\langle\rho, L(\rho) \varphi\rangle=0$ for all $\varphi \in C_{K}^{\infty}\left(R^{d}\right)$.

For each given $\rho \in M_{2}\left(R^{d}\right)$, consider the following stochastic differential equations

$$
\left\{\begin{array}{c}
d X(t)=b(X(t), \rho) d t+\sigma(X(t), \rho) d W(t), \quad t \geq 0 . \\
X(0) \text { has the initial law } \mu_{0}, \mu_{0} \in M_{2}\left(R^{d}\right) .
\end{array}\right.
$$


Under the assumption $(A)$, equation (22) has a unique continuous solution. Let $X_{\rho}$ denote the unique solution of (22). Then the process $X_{\rho}$ is a time homogeneous Markov-Feller process. The associated transition semigroup $\left\{T_{\rho}(t): t \geq 0\right\}$ has the form

$$
T_{\rho}(t) \varphi(x)=\int_{R^{d}} \varphi(y) P_{\rho}(t, x, d y), \quad \varphi \in C_{b}\left(R^{d}\right),
$$

where $P_{\rho}(t, x, B)=P\left(X_{\rho}(t) \in B \mid X_{\rho}(0)=x\right), t \geq 0, x \in R^{d}, B \in \mathbb{B}\left(R^{d}\right)$, is the usual transition function of a Markov process. Let $\mu_{\rho}(t)$ denote the probability law of $X_{\rho}(t)$. Then the associated McKean-Vlasov equation becomes

$$
\left\{\begin{array}{l}
\frac{d}{d t}\left\langle\mu_{\rho}(t), \varphi\right\rangle=\left\langle\mu_{\rho}(t), L(\rho), \varphi\right\rangle, \quad t>0, \forall \varphi \in C_{K}^{\infty}\left(R^{d}\right) \\
\mu_{\rho}(0)=\mu_{0} .
\end{array}\right.
$$

Clearly if $\rho$ is an invariant measure of system (19), then it is also an invariant measure of the diffusion process $X_{\rho}$. This observation suggests that in order to find invariant measures for the nonlinear Markov process defined by equation (19) (which is hard in general) one should search among the invariant measures of the time homogeneous Markov process defined by equation (22) (which is relatively easy in general). With this strategy in mind, we now define for each $\rho \in M_{2}\left(R^{d}\right)$ a subset $\varphi_{\rho}$ of $M_{2}\left(R^{d}\right)$ as following:

$$
\varphi_{\rho}=\left\{Q \in M_{2}\left(R^{d}\right):\langle Q, L(\rho) \varphi\rangle=0 \text { for all } \varphi \in C_{K}^{\infty}\left(R^{d}\right)\right\} .
$$

Proposition 1: The following two conditions are equivalent

(i) $Q \in \varphi_{\rho}$;

(ii) $\int_{R^{d}}\left(T_{\rho}(t) \varphi\right)(x) Q(d x)=\int_{R^{d}} \varphi(x) Q(d x)$ for all $\varphi \in C_{K}^{\infty}\left(R^{d}\right)$.

Proof: $\quad(i) \rightarrow(i i)$. For any $\varphi \in C_{K}^{\infty}$, we have $T_{\rho}(t) \varphi-\varphi=\int_{0}^{t} L(\rho)\left(T_{\rho}(s)\right.$ $\varphi) d s$. Since $T_{\rho}(s) \varphi \in C_{K}^{\infty}$, condition $(i)$ implies

$$
\begin{gathered}
\int_{R^{d}}\left(T_{\rho}(t) \varphi\right)(x) Q(d x)-\int_{R^{d}} \varphi(x) Q(d x) \\
=\left\langle T_{\rho}(t) \varphi, Q\right\rangle-\langle\varphi, Q\rangle \\
=\int_{0}^{t}\left\langle L(\rho)\left(T_{\rho}(s) \varphi\right), Q\right\rangle d s=0 .
\end{gathered}
$$


$(i i) \rightarrow(i)$. For any $\varphi \in C_{K}^{\infty}$, we also have $T_{\rho}(t) \varphi-\varphi=\int_{0}^{t} T_{\rho}(s)(L(\rho) \varphi) d s$, so $(i i)$ implies

It follows that

$$
\int_{0}^{t}\left\langle T_{\rho}(s)(L(\rho) \varphi), Q\right\rangle d s=0
$$

$$
\langle L(\rho) \varphi, Q\rangle=\lim _{t \rightarrow 0} \frac{1}{t} \int_{0}^{t}\left\langle T_{\rho}(s)(L(\rho) \varphi), Q\right\rangle d s=0 .
$$

For each positive integer $N>1$, let $Q_{N}^{\rho}$ be defined by

$$
Q_{N}^{\rho}=\frac{1}{N} \int_{0}^{N} \mu_{\rho}(t) d t .
$$

Proposition 2: Suppose $Q_{\rho}$ is a limit point of $\left\{Q_{N}^{\rho}\right\}$. Then $Q_{\rho} \in \varphi_{\rho}$.

Proof: Let $Q_{\rho} \in M_{2}\left(R^{d}\right)$ such that $\left\{Q_{N_{k}}^{\rho}\right\}$ converges weakly to $Q_{\rho}$ as $k$ goes to infinity, where $\left\{Q_{N_{k}}^{\rho}\right\}$ is a subsequence of $\left\{Q_{N}^{\rho}\right\}$. As in (22) we let $\mu_{0}(d x)$ be the initial distribution of the process $X_{\rho}(0)$. Since $X_{\rho}$ is a Feller process, $T_{\rho}(t) \varphi \in C_{b}\left(R^{d}\right)$ for each $\varphi \in C_{b}\left(R^{d}\right)$. Thus we have

$$
\begin{gathered}
\int_{R_{d}}\left(T_{\rho}(t) \varphi\right)(x) Q_{\rho}(d x)=\lim _{k \rightarrow \infty} \int_{R^{d}}\left(T_{\rho}(t) \varphi\right)(x) Q_{N_{k}}(d x) \\
=\lim _{k \rightarrow \infty} \frac{1}{N_{k}} \int_{0}^{N_{k}} E\left[\left(T_{\rho}(t) \varphi\right)\left(X_{\rho}(s)\right)\right] d s \\
=\lim _{k \rightarrow \infty} \frac{1}{N_{k}} \int_{0}^{N_{k}} \int_{R^{d}}\left(T_{\rho}(t+s) \varphi\right)(x) \mu_{0}(d x) d s \\
=\lim _{k \rightarrow \infty} \frac{1}{N_{k}} \int_{t}^{t+N_{k}} \int_{R^{d}}\left(T_{\rho}(s) \varphi\right)(x) \mu_{0}(d x) d s \\
=\lim _{k \rightarrow \infty} \frac{t+N_{k}}{N_{k}} \cdot \frac{1}{t+N_{k}} \int_{0}^{t+N_{k}} \int_{R^{d}}\left(T_{\rho}(s) \varphi\right)(x) \mu_{0}(d x) d s \\
-\lim _{k \rightarrow \infty} \frac{1}{N_{k}} \int_{0}^{t} \int_{R^{d}}\left(T_{\rho}(s) \varphi\right)(s) \mu_{0}(d x) d s
\end{gathered}
$$




$$
\begin{gathered}
=\lim _{k \rightarrow \infty} \frac{1}{N_{k}} \int_{0}^{N_{k}} \int_{R^{d}}\left(T_{\rho}(s) \varphi\right)(x) \mu_{0}(d x) d s \\
=\lim _{k \rightarrow \infty} \frac{1}{N_{k}} \int_{0}^{N_{k}} E \varphi\left(X_{\rho}(s)\right) d s \\
=\lim _{k \rightarrow \infty} \int_{R^{d}} \varphi(x) Q_{N_{k}}^{\rho}(d x) \\
=\int_{R^{d}} \varphi(x) Q_{\rho}(d x) .
\end{gathered}
$$

This shows that $Q_{\rho}$ is an invariant measure corresponding to $X_{\rho}$. Thus, by proposition $1, Q_{\rho}$ satisfies $\left\langle Q_{\rho}, L(\rho) \varphi\right\rangle=0$ for all $\varphi \in C_{K}^{\infty}\left(R^{d}\right)$, and so $Q_{\rho} \in \varphi_{\rho}$.

The following theorem gives a general result on the existence and uniqueness of invariant measures of the nonlinear Markov processes described by equation (19).

Theorem 2: $\quad$ Suppose that there exists a nonempty closed subset $\Xi$ of $M_{2}\left(R^{d}\right)$ such that the following three conditions are satisfied:

(a) for each $\rho \in \Xi, \varphi_{\rho} \subset \Xi$,

(b) for each $\rho \in \Xi$, $\sup _{t} \geq 0 \frac{1}{t} \int_{0}^{t} E\left|X_{\rho}(s)\right|^{2} d s<\infty$,

(c) there exists a constant $c \in(0,1)$ such that for any $p, q, P$ and $Q$ in $\Xi$, we have

$$
\lim _{t \rightarrow \infty} \rho_{2}\left(\mu_{p}(t ; P), \mu_{q}(t ; Q)\right) \leq c \rho_{2}(p, q),
$$

where $\mu_{\rho}\left(t ; \mu_{0}\right)\left(\rho=p, q ; \mu_{0}=P, Q\right)$ denotes the probability law of $X_{\rho}(t)$ of $(22)$ with initial condition $\mu_{\rho}\left(0 ; \mu_{0}\right)=\mu_{0}$.

Then the nonlinear Markov process $X$ determined by (19) has an invariant measure.

Before proving this theorem we first state a generalized Banach fixedpoint theorem for multivalued maps on metric spaces (see, for example, [27]).

Definition 2: $\quad$ Let $(X, d)$ be a metric space. If $A, B$ are two subsets of $X$, then the Hausdorff matrix $H(A, B)$ between them is defined as 


$$
H(A, B)=\max \left\{\sup _{a \in A} d(a, B), \sup _{b \in B} d(b, A)\right\}
$$

where $d(a, B)=i n f_{b \in B} d(a, b)$ is the distance of the point $a$ from the set $B$.

Theorem (Generalized Banach fixed-point theorem for multivalued maps):

Let $(X, d)$ be a metric space and $K$ be a subset of $X$. Let $\Gamma: K \rightarrow 2^{K}\left(2^{K}\right.$ denotes the collection of all subsets of $K$ ) be a multivalued map. Suppose that

(i) $K$ is nonempty and closed;

(ii) for each $x \in K$, the set $\Gamma(x)$ is nonempty and closed;

(iii) there exists a constant $c \in(0,1)$ such that the condition

$$
H(\Gamma(x), \Gamma(y)) \leq c d(x, y)
$$

is satisfied for all $x, y \in K$.

Then $\Gamma$ has a fixed point $x^{*}$, that is, $x^{*} \in \Gamma\left(x^{*}\right)$.

Proof of theorem 2: Let $\Gamma: \Xi \rightarrow 2 \Xi$ be the multivalued map defined by

$$
\Gamma(\rho)=\varphi_{\rho} .
$$

Then by assumption (a) the map $\Gamma$ is well defined. Suppose that the map $\Gamma$ has a fixed point $\rho^{*}$, that is, $\rho^{*} \in \Gamma\left(\rho^{*}\right)$. Then, by the definition of $\varphi_{\rho}$, this fixed point $\rho^{*}$ must satisfy the equation $\left\langle\rho^{*}, L\left(\rho^{*}\right) \varphi\right\rangle=0$ for all $\varphi \in C_{K}^{\infty}\left(R^{d}\right)$. Thus $\rho^{*}$ is an invariant measure of system (19) and so the proof of theorem 2 will be finished. Since $\Gamma$ is defined on the nonempty and closed subset $\Xi$ of the metric space $\left(M_{2}\left(R^{d}\right), \rho_{2}\right)$, we apply the generalized Banach fixed-point theorem to this multivalued map. Thus we need to check if the conditions (ii) and (iii) of generalized Banach fixed-point theorem are satisfied.

On condition (ii): We first show that, for each $\rho \in \Xi$, the set $\Gamma(\rho)$ is nonempty. Let $Q_{N}^{\rho}$ be defined as in (29). Then according to proposition 2 it suffices to show that $\left\{Q_{N}^{\rho}\right\}$ is relatively compact. By the assumption $(b)$ of theorem 2 we have

$$
\int_{R^{d}}|x|^{2} Q_{N}^{\rho}(d x)=\frac{1}{N} \int_{0}^{N} E\left|X_{\rho}(s)\right|^{2} d s<\infty, \quad \forall N \geq 1 .
$$

For each $\epsilon>0$, Chebyshev's inequality then implies that there is an $R_{\epsilon}>0$ such that

$$
Q_{N}^{\rho}\left(\left\{x:|x|^{2}>R_{\epsilon}\right\}\right) \leq \frac{\int_{R^{d}}|x|^{2} Q_{N}^{\rho}(d x)}{R_{\epsilon}} \leq \epsilon, \quad \forall N \geq 1
$$


Thus, for each $\epsilon>0$, there is a compact set $K_{\epsilon}=\left\{x:|x|^{p} \leq R_{\epsilon}\right\}$ such that

$$
\inf _{N \geq 1} Q_{N}^{\rho}\left(K_{\epsilon}\right)>1-\epsilon
$$

Thus $\left\{Q_{N}^{\rho}\right\}$ is relatively compact according to Prohorov's theorem.

Next we show that the set $\varphi_{\rho}$ is closed in $\Xi$ for each $\rho \in \Xi$. Let $\left\{Q_{n}\right\}$ be a sequence of measures in $\varphi_{\rho}$ such that $Q_{n}$ converges to some $Q \in M_{2}\left(R^{d}\right)$ in the metric space $\left(M_{2}\left(R^{d}\right), \rho_{2}\right)$. Then $Q_{n}$ converges to $Q$ weakly in $M_{2}\left(R^{d}\right)$. Since for any $\varphi \in C_{K}^{\infty}$, the function $L(\rho) \varphi$ is continuous and bounded, we have

$$
\langle Q, L(\rho) \varphi\rangle=\lim _{n \rightarrow \infty}\left\langle Q_{n}, L(\rho) \varphi\right\rangle=0 .
$$

Thus $Q \in \varphi_{\rho}$ and so $\varphi_{\rho}$ is closed in $M_{2}\left(R^{d}\right)$. Moreover, $Q \in \Xi$ because, by assumption ( $a$ ), each $Q_{n}$ belongs to $\Xi$, and $\Xi$ is closed. Thus $\varphi_{\rho}$ is a closed subset of $\Xi$.

On condition (iii): We now show that the generalized contraction condition

$$
H(\Gamma(p), \Gamma(q)) \leq c \rho_{2}(p, q)
$$

is satisfies for all $p, q \in \Xi$ and a fixed $c \in(0,1)$.

Let $P \in \Gamma(p)$ and $Q \in \Gamma(q)$ be arbitrary two elements. Let $X_{p}(\cdot ; P)$ and $X_{q}(\cdot ; Q)$ be the unique solution of equation (22) with $\rho$ replaced by $p$ and $q$, and $\mu_{0}$ replaced by $P$ and $Q$, respectively. The probability law of $X_{p}(t ; P)$ and $X_{q}(t ; Q)$ will be denoted by $\mu_{p}(t ; P)$ and $\mu_{q}(t ; Q)$, respectively. Since $P \in \varphi_{p}$ and $Q \in \Psi_{q}$, they are invariant measures of the corresponding processes $X_{p}(t ; P)$ and $X_{q}(t ; Q)$, that is, $P=\mu_{p}(t ; P)$ and $Q=\mu_{p}(t ; Q)$ for all $t \geq 0$. Thus assumption $(c)$ implies

$$
\rho_{2}(P, Q)=\lim _{t \rightarrow \infty} \rho_{2}\left(\mu_{p}(t ; P), \mu_{q}(t ; Q)\right) \leq c \rho_{2}(p, q) .
$$

It follows from (38) that

$$
H(\Gamma(p), \Gamma(q))=\max \left\{\sup _{P \in \mathcal{\varphi}_{q} Q \in \mathcal{I}_{q}} \inf _{2}(P, Q), \sup _{Q \in \mathcal{S}_{p} P \in \mathcal{I}_{p}} \inf _{2}(Q, P)\right\} \leq c \rho_{2}(p, q) .
$$

This completes the proof of theorem 2 . 


\section{EXISTENCE AND UNIQUENESS OF INVARIANT MEASURES}

We are now going to apply the general result in section 3 to our original equation (1). To this end we consider the following stochastic differential equation

$$
\left\{\begin{array}{l}
d X(t)=[-A X(t)+f(X(t), \rho)] d t+d W(t), \quad t \geq 0 . \\
X(0) \text { has the initial law } \mu_{0} \in M_{2}\left(R^{d}\right)
\end{array}\right.
$$

where $\rho \in M_{2}\left(R^{d}\right)$.

Proposition 3: Assume the conditions of theorem 1 hold. Then,

(a) for each $\rho \in \Xi$, equation (40) has a unique solution $X_{\rho}$ which can be written as

$$
X_{\rho}(t)=S(t) X_{\rho}(0)+\int_{0}^{t} S(t-s) f\left(X_{\rho}(s), \rho\right) d s+\int_{0}^{t} S(t-s) d W(s)
$$

where $S(t)=\exp (-A t$ is the semigroup generated by $A$;

(b) if the two constants $\omega$ and $l$ in assumptions (A1) and (A2) satisfy $\omega^{2}>3 l$, then we have that $\sup _{t \geq 0} E\left|X_{\rho}(t)\right|^{2} \leq \frac{\omega^{2} \alpha}{\omega^{2}-3 l}<+\infty$ holds true for any $\rho \in M_{2}\left(R^{d}\right)$, where $\alpha$ is a finite positive constant depending on $\rho$.

Proof: (a) For a given $\rho \in \Xi$, equation (40) is an ordinary stochastic differential equation. Thus the Lipschitz and linear growth conditions, as specified by $(A 1)$ and $(A 2)$, ensure that the same Picard iteration scheme used in the proof of theorem 1 will result a unique solution $X_{\rho}$ of the form in $(a)$.

(b) For $t>0$, using $(a+b+c)^{2} \leq 3\left(|a|^{2}+|b|^{2}+|c|^{2}\right)$ and Hölder's inequality, we have

$$
\begin{gathered}
E\left|X_{\rho}(t)\right|^{2} \leq 3\|S(t)\|^{2} E\left|X_{\rho}(0)\right|^{2}+3 E\left|\int_{0}^{t} S(t-s) d W(s)\right|^{2} \\
+3 E\left|\int_{0}^{t} S(t-s) f\left(X_{\rho}(s), \rho\right) d s\right|^{2} \\
\leq 3 E\left|X_{\rho}(0)\right|^{2}+3 \int_{0}^{t}\|S(s)\|^{2} d s \\
+\int_{0}^{t}\|S(s)\| d s E \int_{0}^{t}\|S(t-s)\|\left|f\left(X_{\rho}(s), \rho\right)\right|^{2} d s
\end{gathered}
$$


Since $\int_{0}^{t}\|S(s)\| d s \leq \frac{1}{\omega}[1-\exp (-\omega t)]$ and $\left|f\left(X_{\rho}(s), \rho\right)\right|^{2} \leq l\left(1+\left|X_{\rho}(s)\right|^{2}+\right.$ $\left.\|\rho\|_{2}^{2}\right)$, (41) can be reduced to

$$
E\left|X_{\rho}(t)\right|^{2} \leq \alpha+\frac{3 l}{\omega} \int_{0}^{t} \exp \{-\omega(t-s)\} E\left|X_{\rho}(s)\right|^{2} d s
$$

where $\alpha=3 E\left|X_{\rho}(0)\right|^{2}+\frac{3}{\omega}+\frac{3 l}{\omega^{2}}\left(1+\|\rho\|_{2}^{2}\right)$. Denote $\Phi(t)=\exp (\omega t) E\left|X_{\rho}(t)\right|^{2}$ and $f(t)=\exp (\omega t) \alpha$. Then (42) has the form of

and so the Gronwall's inequality gives

$$
\Phi(t)=f(t)+\frac{3 l}{\omega} \int_{0}^{t} \Phi(s) d s
$$

Thus for $\omega^{2}>3 l$, we have

$$
\Phi(t) \leq f(t)+\frac{3 l}{\omega} \int_{0}^{t} \exp \left\{\frac{3 l}{\omega}(t-s)\right\} f(s) d s .
$$

$$
\begin{gathered}
\sup _{t \geq 0} E\left|X_{\rho}(t)\right|^{2} \leq \alpha+\alpha \frac{3 l}{\omega} \sup _{t \geq 0} \int_{0}^{t} \exp \left\{-\left(\omega-\frac{3 l}{\omega}\right)(t-s)\right\} d s \\
\leq \alpha+\alpha \frac{3 l}{\omega^{2}-3 l} \sup _{t \geq 0}\left[1-\exp \left\{-\left(\omega-\frac{3 l}{\omega}\right) t\right\}\right] \\
\leq \frac{\omega^{2} \alpha}{\omega^{2}-3 l}<\infty
\end{gathered}
$$

Let $\mu_{\rho}(t)$ denote the probability law of the unique solution $X_{\rho}$ of $(40)$ and define $Q_{N}^{\rho}=\frac{1}{N} \int_{0}^{N} \mu_{\rho}(t) d t$ for each $N \geq 1$. Let $\varphi_{\rho}$ be the subset of $M_{2}\left(R^{d}\right)$ defined by (25) with $L(\rho) \varphi(x)=\frac{1}{2} \Delta \varphi(x)-(A x-f(x, \rho), \nabla \varphi(x)(x))$, where $\Delta$ and $\nabla$ denote the Laplacian and gradient operator, respectively. Then the sequence of probability measures $\left\{Q_{N}^{\rho}\right\}$ is relatively compact in $M\left(R^{d}\right)$ due to the result (b) of proposition 3 and $\varphi_{\rho}$ is the set of all limit points of $\left\{Q_{N}^{\rho}\right\}$. For a given $s>0$, let $\Xi_{s}$ be the subset of $M_{2}\left(R^{d}\right)$ defined by

$$
\Xi_{s}=\left\{\mu \in M_{2}\left(R^{d}\right): \int_{R^{d}}|x|^{2} \mu(d x) \leq s\right\} .
$$

Then $\Xi_{s}$ is a nonempty closed subset of $M_{2}\left(R^{d}\right)$.

Proposition 4: Suppose that the two constants $\omega$ and $l$ in assumptions (A1) and (A2) satisfy the inequality $\omega^{2}>6 l$. Then there exists a real $s>0$ such that for any $\rho \in \Xi_{s}$, we have $\varphi_{\rho} \subset \Xi_{s}$. 
Proof: Let $Q_{\rho} \in \varphi_{\rho}$ for some $\rho \in M_{2}\left(R^{d}\right)$. Then there exists a subsequence $\left\{Q_{N_{k}}^{\rho}\right\}$ of $\left\{Q_{N}^{\rho}\right\}$ such that $Q_{N_{k}}^{\rho}$ converges to $Q_{\rho}$ as $k \rightarrow \infty$. Thus for each positive integer $M \geq 1$, by proposition $3(b)$, we have

$$
\begin{gathered}
\int_{R^{d}}\left(|x|^{2} \wedge M\right) Q_{\rho}(d x)=\lim _{k \rightarrow \infty} \int_{R^{d}}\left(|x|^{2} \wedge M\right) Q_{N_{k}}^{\rho}(d x) \\
=\lim _{k \rightarrow \infty} \frac{1}{N_{k}} \int_{0}^{N_{k}} \int_{R^{d}}\left(|x|^{2} \wedge M\right) \mu_{\rho}(t)(d x) d t \\
\leq \lim _{k \rightarrow \infty} \frac{1}{N_{k}} \int_{0}^{N_{k}} E\left|X_{\rho}(t)\right|^{2} d t \\
\leq \frac{\omega^{2} \alpha}{\omega^{2}-3 l},
\end{gathered}
$$

where $\alpha=3 E\left|X_{\rho}(0)\right|^{2}+\frac{3}{\omega}+\frac{3 l}{\omega^{2}}\left(1+\|\rho\|_{2}^{2}\right)$. Letting $M$ go to infinity we have that

$$
\int_{R^{d}}|x|^{2} Q_{\rho}(d x) \leq \frac{\omega^{2} \alpha}{\omega^{2}-3 l} .
$$

Let $s=\frac{3 \omega^{2} E\left|X_{\rho}(0)\right|^{2}+3 \omega+3 l}{\omega^{2}-6 l}$, then it is easy to check that the right-hand side of (48) satisfies

$$
\frac{\omega^{2} \alpha}{\omega^{2}-3 l} \leq s \text { if }\|\rho\|_{2}^{2} \leq s .
$$

Thus $Q_{\rho} \in \Xi_{s}$ for any $\rho \in \Xi_{s}$ and so the proof is completed.

Let $X_{p}(\cdot ; P)$ and $X_{q}(\cdot ; Q)$ be the unique solution of the equation (40) with $\rho$ replaced by $p$ and $q$, and $\mu_{0}$ replaced by $P$ and $Q$, respectively. The probability law of $X_{p}(t ; P)$ and $X_{q}(t ; Q)$ will be denoted by $\mu_{p}(t ; P)$ and $\mu_{q}(t ; Q)$, respectively.

Proposition 5: If the two constants $\omega$ and $k$ in assumptions (A1) and (A2) satisfy the inequality $\omega^{2}>4 k$, then there exists a constant $c \in(0,1)$ such that

$$
\lim _{t \rightarrow \infty} \rho_{2}\left(\mu_{p}(t ; P), \mu_{q}(t ; Q)\right) \leq c \rho_{2}(p, q)
$$

for any $p, q, P, Q$ in $\Xi_{s}$.

Proof: According to the definition of the metric $\rho_{2}$, it suffices to show 
that

$$
\lim _{t \rightarrow \infty} E\left|X_{p}(t ; P)-X_{q}(t ; Q)\right|^{2} \leq c^{2} \rho_{2}^{2}(p, q)
$$

for any $p, q, P$ and $Q$ in $\Xi_{s}$. By definition,

$$
X_{p}(t ; P)=S(t) X_{p}(0 ; P)+\int_{0}^{t} S(t-s) f\left(X_{p}(s ; P), p\right) d s+\int_{0}^{t} S(t-s) d W(s)
$$

and

$$
X_{q}(t ; Q)=S(t) X_{q}(0 ; Q)+\int_{0}^{t} S(t-s) f\left(X_{q}(s ; Q), q\right) d s+\int_{0}^{t} S(t-s) d W(s) .
$$

Using Hölder's inequality and assumption $(A 2)$ we have

$$
\begin{gathered}
E\left|X_{p}(t ; P)-X_{q}(t ; Q)\right|^{2} \leq 2\|S(t)\|^{2} E\left|X_{p}(0 ; P)-X_{q}(0 ; Q)\right|^{2} \\
+2 E \int_{0}^{t}\|S(t)\| d s \int_{0}^{t}\|S(t-s)\|\left|f\left(X_{p}(s ; P), p\right)-f\left(X_{q}(t ; Q), q\right)\right|^{2} d s \\
\leq 2\|S(t)\|^{2} E\left|X_{p}(0 ; P)-X_{q}(0 ; Q)\right|^{2}+2 k \int_{0}^{t}\|S(t)\| d s \\
\int_{0}^{t}\|S(t-s)\|\left(E\left|X_{p}(s ; P)-X_{q}(s ; Q)\right|^{2}+\rho_{2}^{2}(p, q)\right) d s .
\end{gathered}
$$

Using assumption (A1) the expression (52) can be reduced to

$$
\begin{gathered}
E\left|X_{p}(t ; P)-X_{q}(t ; Q)\right|^{2} \leq \exp (-\omega t) f(t) \\
+\frac{2 k}{\omega} \int_{0}^{t} \exp \{-\omega(t-s)\} E\left|X_{p}(s ; P)-X_{q}(s ; Q)\right|^{2} d s,
\end{gathered}
$$

where $f(t)=2 E\left|X_{p}(0 ; P)-X_{q}(0 ; Q)\right|^{2}+\frac{2 k}{\omega^{2}} \exp (\omega t) \rho_{2}^{2}(p, q)$.

Denote $\Phi(t)=\exp (\omega t) E\left|X_{p}(t ; P)-X_{q}(t ; Q)\right|^{2}$, then (53) can be rewritten as

and so Gronwall's equality gives

$$
\Phi(t) \leq f(t)+\frac{2 k}{\omega} \int_{0}^{t} \Phi(s) d s
$$

$$
\Phi(t) \leq f(t)+\frac{2 k}{\omega} \int_{0}^{t} \exp \left\{\frac{2 k}{\omega}(t-s)\right\} f(s) d s
$$

Thus

$$
E\left|X_{p}(t ; P)-X_{q}(t ; Q)\right|^{2} \leq \exp (-\omega t) f(t)
$$




$$
+\frac{2 k}{\omega} \exp (-\omega t) \int_{0}^{t} \exp \left\{\frac{2 k}{\omega}(t-s)\right\} f(s) d s
$$

In the limit of $t \rightarrow \infty$, the first term on the right-hand side of (56) becomes

$$
\begin{gathered}
\lim _{t \rightarrow \infty} \exp (-\omega t) f(t) \\
=\lim _{t \rightarrow \infty} 2 \exp (-\omega t) E\left|X_{p}(0 ; P)-X_{q}(0 ; Q)\right|^{2}+\frac{2 k}{\omega^{2}} \rho_{2}^{2}(p, q) \\
=\frac{2 k}{\omega^{2}} \rho_{2}^{2}(p, q),
\end{gathered}
$$

and the second term on the right-hand side of (56) becomes

$$
\begin{gathered}
\lim _{t \rightarrow \infty} \frac{2 k}{\omega} \exp (-\omega t) \int_{0}^{t} \exp \left\{\frac{2 k}{\omega}(t-s)\right\} f(s) d s \\
\leq \lim _{t \rightarrow \infty} 2 E\left|X_{p}(0 ; P)-X_{q}(0 ; Q)\right|^{2}\left[\exp \left\{-\left(\omega-\frac{2 k}{\omega}\right) t\right\}-\exp (-\omega t)\right] \\
+\lim _{t \rightarrow \infty}\left(\frac{(2 k)^{2}}{\omega^{3}}\right)\left(\frac{1}{\omega-\frac{2 k}{\omega}}\right) \rho_{2}^{2}(p, q)\left[1-\exp \left\{-\left(\omega-\frac{2 k}{\omega}\right) t\right\}\right] \\
=\left(\frac{(2 k)^{2}}{\omega^{3}}\right)\left(\frac{1}{\omega-\frac{2 k}{\omega}}\right) \rho_{2}^{2}(p, q) .
\end{gathered}
$$

Thus we have

$$
\begin{gathered}
\lim _{t \rightarrow \infty} E\left|X_{p}(t ; P)-X_{q}(t ; Q)\right|^{2} \leq\left[\frac{2 k}{\omega^{2}}+\frac{(2 k)^{2}}{\omega^{3}}\left(\frac{1}{\omega-\frac{2 k}{\omega}}\right)\right] \rho_{2}^{2}(p, q) \\
=\frac{2 k}{\omega^{2}-2 k} \rho_{2}^{2}(p, q) .
\end{gathered}
$$

Let $c^{2}=\frac{2 k}{\omega^{2}-2 k}$. Then the assumption $\omega^{2}>4 k$ implies $c \in(0,1)$ and so the proof is completed.

We are now ready to state the main theorem of this paper:

Theorem 3: $\quad$ Let the conditions $(A 1)$ and $(A 2)$ be satisfied. If the three constants $\omega, l$ and $k$ in assumptions $(A 1)$ and $(A 2)$ satisfy the condition

$$
\omega^{2}>\max (6 l, 4 k)
$$

then the nonlinear Markov process $X$ determined by Itô equation (1) has a unique invariant measure for any $X(0) \in L_{2}\left(\Omega, \mathcal{F}_{0}, P ; R^{d}\right)$.

Proof (Existence): We use theorem 2 of section 3. For each $r>0$, let $\Xi_{r}$ be the subset of $M_{2}\left(R^{d}\right)$ defined in (46). Then, by proposition 4 , there exists a positive number $s$ such that the corresponding set $\Xi_{s}$ satisfies condition $(a)$ of 
theorem 2. Moreover, by proposition $3(b)$, condition (b) of theorem 2 holds. Finally, by proposition 5, condition $(c)$ of theorem 2 is also satisfied. Hence existence part of the theorem is true.

(Uniqueness): It is important to note that for multivalued maps on metric spaces the Banach fixed point theorem does not, in general, imply uniqueness. Thus to seek uniqueness we have to use other methods.

Suppose that $\mu$ and $\nu$ be two arbitrary invariant measures of $X$. We show $\rho_{2}(\mu, \nu)=0$. Let $X$ denote the unique solution of the equation (1) and let $\mu(t)$ be the probability law of $X(t)$. For $t \geq 0$, let $U(t)$ denote the nonlinear semigroup on $M_{2}\left(R^{d}\right)$ defined by $U(t) \mu_{0}=\mu(t)$ for any $\mu_{0} \in M_{2}\left(R^{d}\right)$. Recall that a probability measure $\rho \in M_{2}\left(R^{d}\right)$ is an invariant measure for the nonlinear Markov process $X$ if $U(t) \rho=\rho$ for all $t \geq 0$. Let $X(t ; x)$ and $X(t ; y)$ denote the unique solution of (1) with initial data $X(0 ; x)=x$ and $X(0 ; y)=y$, respectively. The corresponding distribution will be denoted by $\mu_{x}(t)$ and $\mu_{y}(t)$, respectively. Since

$$
\begin{gathered}
\rho_{2}^{2}(\mu, \nu)=\rho_{2}^{2}(U(t) \mu, U(t) \nu) \\
\leq \int_{R^{d} \times R^{d}} E\left(|X(t ; x)-X(t ; y)|^{2} \wedge 1\right)(\mu \times \nu)(d x, d y)
\end{gathered}
$$

it suffices to show that

$$
E\left(|X(t ; x)-X(t ; y)|^{2}\right) \rightarrow 0
$$

for any $x, y \in R^{d}$.

By assumptions $(A 1)$ and $(A 2)$

$$
\begin{gathered}
E|X(t ; x)-X(t ; y)|^{2} \leq 2\|S(t)\|^{2}|x-y|^{2} \\
+2 E\left|\int_{0}^{t} S(t-s)\left(f\left(X(s ; x), \mu_{x}(s)\right)-f\left(X(s ; y), \mu_{y}(s)\right)\right) d s\right|^{2} \\
\leq 2\|S(t)\|^{2}|x-y|^{2}+2 \int_{0}^{t}\|S(s)\| d s E \int_{0}^{t}\|S(t-s)\| \\
\left.\mid f\left(X(s ; x), \mu_{x}(s)\right)-f\left(X(s ; y), \mu_{y}(s)\right)\right)\left.\right|^{2} d s \\
\leq 2 \exp (-\omega t)|x-y|^{2}+\frac{2 k}{\omega}[1-\exp (-2 \omega t)] \int_{0}^{t} \exp \{-\omega(t-s)\}
\end{gathered}
$$




$$
\begin{gathered}
\left(E|X(s ; x)-X(s ; y)|^{2}+\rho_{2}^{2}\left(\mu_{x}(s), \mu_{y}(s)\right)\right) d s \\
\leq 2 \exp (-\omega t)|x-y|^{2}+\frac{4 k}{\omega}[1-\exp (-2 \omega t)] \int_{0}^{t} \exp \{-\omega(t-s)\} \\
E|X(s ; x)-X(s ; y)|^{2} d s .
\end{gathered}
$$

Denote $\Phi(t)=\exp (\omega t) E|X(t ; x)-X(t ; y)|^{2}$, then (62) can be written as

Gronwall's inequality then yields

$$
\Phi(t) \leq 2|x-y|^{2}+\frac{4 k}{\omega} \int_{0}^{t} \Phi(s) d s .
$$

$$
\Phi(t) \leq 2|x-y|^{2} \exp \left\{\frac{4 k}{\omega} t\right\}
$$

that is,

$$
E|X(t ; x)-X(t ; y)|^{2} \leq 2|x-y|^{2} \exp \left\{-\left(\omega-\frac{4 k}{\omega}\right) t\right\}
$$

By assumption, $\omega-\frac{4 k}{\omega}$ is strictly positive, and so the right-hand side of (65) tends to zero as $t \rightarrow \infty$. It follows from the definition (3) that

$$
\rho_{2}\left(U(t) \delta_{x}, U(t) \delta_{y}\right) \leq E|X(t ; x)-X(t ; y)|^{2} \rightarrow 0, \text { as } t \rightarrow \infty
$$

This completes the proof of uniqueness.

Example: Consider the following equation in $R^{1}$ :

$$
d X(t)=[-\alpha X(t)+E(X(t))] d t+d W(t), \quad t \geq 0
$$

where $W$ is a standard one-dimensional Wiener process; $\alpha$ is a positive constant; $E(X(t))$ is the mean of $X(t)$. In other words, the function $f(x, \mu)$ in (1) now assumes the simple form $f(x, \mu)=\int_{R^{1}} x \mu(d x)$. It is easy to verify that this model satisfies conditions $(A 1)$ and $(A 2)$ with $k=l=1$ and $\omega=\alpha$. According to theorem 3, system (67) will have a unique invariant measure if $\alpha$ satisfies $\alpha^{2}>6$ which is true as we will show below. But the following exposition also indicates that this condition is not a necessary condition for the existence of a unique invariant measure.

Since (67) is a gradient system, the corresponding invariant measures have the form 


$$
P_{m}(d x)=\frac{1}{Z} \exp \left\{-\left(\alpha x^{2}-2 m x\right)\right\} d x,
$$

where $Z$ is the normalizing constant which ensures that $P_{m}(d x)$ is a probability measure, and the constant $m$ must satisfy the self-consistence equation

$$
m=\int_{R^{1}} x P_{m}(d x) .
$$

By a simple algebraic manipulation, it is easy to see that $P_{m}(d x)$ is a Gaussian measure on $R^{1}$ with mean $\frac{m}{\alpha}$ and variance $\frac{\alpha}{2}$. Thus the self-consistent equation reduces to the algebraic equation

$$
m=\frac{m}{\alpha} .
$$

It follows from (70) that system (67) will have a unique invariant measure (which is a zero-mean-Gaussian measure) if $\alpha \neq 1$ and will have infinitely many invariant measures if $\alpha=1$. This shows that the condition given by theorem 3 is only a sufficient condition.

It is interesting to point out that even for this simple model of a nonlinear Markov process, its long time behavior is not trivial. For example, for $\alpha \neq 1$, although system (67) has a unique invariant measure, the distribution of the process at time $t$ will not always converge to it as $t$ becomes large. This can easily be seen from the following calculation.

Equation (67) can be rewritten as

$$
X(t)=X(0)-\int_{0}^{t}[\alpha X(s)-E(X(s))] d s+W(t) .
$$

Let $m(t)$ denote the mean of $X(t)$ with initial data $m(0)=m_{0}$. Then by taking the expectation on both sides of (71) we have

$$
m(t)=m_{0}+\int_{0}^{t}(1-\alpha) m(s) d s,
$$

and therefore $m(t)$ satisfies the equation

$$
\left\{\begin{array}{c}
\frac{d m(t)}{d t}=(1-\alpha) m(t) \\
m(0)=m_{0},
\end{array}\right.
$$

with the solution $m(t)=m_{0} \exp (1-\alpha) t$. Thus for $0<\alpha<1, m(t)$ does not 
converge to $m_{0}$, which means that if we start system (67) from any initial measure other than the invariant measure then the corresponding distribution will never converge to the invariant measure. On the other hand, if $\alpha$ satisfies the condition of theorem 3 , that is $\alpha>\sqrt{6}$, then the mean $m(t)$ will always converge to 0 , which is the mean of the unique invariant measure.

\section{REFERENCES}

[1] Ahmed, N.U., Semigroup Theory with Applications to Systems and Control, Pitman Research Notes in Mathematics 246, Longman Scientific and Technical, UK, John Wiley \& Sons, USA 1991.

[2] Ahmed, N.U., Stability of limit measures induced by stochastic differential equations on Hilbert space, Computers Math. Appl. 22:8 (1991), 41-48.

[3] Beneš, V.E., Finite regular invariant measures for Feller processes, J. Appl. Prob. 5 (1968), 203-209.

[4] Chow, P.L., Stability of stochastic evolution equations, J. Math. Anal. and Appl. 89 (1982), 400-419.

[5] Chow, P.L., Expectation functionals associated with some stochastic evolution equations, Lecture Notes in Mathematics 1236 (edited by G. DaPrato and L. Tubaro), Springer-Verlag (1987), 40-56.

[6] Dawson, D.A., Critical dynamics and fluctuations for a mean-field model of cooperative behavior, J. Stat. Phys. 31 (1983), 29-85.

[7] Dawson, D.A. and Gärtner, J., Large deviations, free energy functional and quasipotential for a mean-field model of interacting diffusions, Memoirs of the American Mathematical Society 398 (1989).

[8] Ding, X., Diffusion processes with random interactions, Ph.D. Thesis, Department of Mathematics and Statistics, Carleton University 1993.

[9] Dobrushin, R.L., Prescribing a system of random variables by conditional distributions, Theory Prob. Appl. 15 (1970), 458-486.

[10] Echeverria, P., A criterion for invariant measures of Markov processes, Z. Wahrsch. vedrw. Gebiete 61 (1982), 1-16.

[11] Ethier, S.N. and Kurtz, T.G., Markov Process: Characterization and Convergence, John Wiley \& Sons 1986.

[12] Funaki, T., A certain class of diffusion processes associated with nonlinear parabolic equations, Prob. Theory and Rel. Fields 67 (1984), 331-348. 
[13] Gärtner, J., On the McKean-Vlasov Limit for Interacting Diffusions I and II, Akademie Der Wissenschaften Der DDR, Karl-Weierstrass-Institute Für Mathematik, Berlin 1986.

[14] Ichikawa, A., Stability of semilinear stochastic evolution equations, J. Math. Anal. Appl. 90 (12), 12-44.

[15] Ichikawa, A., Semilinear stochastic evolution equations. Boundedness, stability and invariant measure, Stochastics 12 (1984), 1-39.

[16] Ikeda, N. and Watanabe, S., Stochastic Differential Equations and Diffusion Processes, North Holland/Kodansha 1989. Second edition.

[17] Maslowski, B., Uniqueness and stability of invariant measures for stochastic differential equations in Hilbert spaces, Stochastic and Stochastic Reports 28 (1989), 85-114.

[18] McKean, H.P., A class of Markov processes associated with nonlinear parabolic equations, Proc. NAS 56 (1966), 1907-1911.

[19] Miyahara, Y., Invariant measures of ultimately bounded stochastic processes, Nagoya Math. J. 49 (1973), 149-153.

[20] DaPrato, G. and Zabczyk, J., Stochastic Differential Equations in Infinite Dimensions, Encyclopedia of Mathematics and Its Applications, Cambridge University Press 1992.

[21] Shigekawa, I., Existence of invariant measures of diffusions on an abstract Wiener space, J. Math. 24 (1987).

[22] Tamura, Y., On asymptotic behaviors of the solution of a nonlinear diffusion equation, J. Fac. Sci. Univ. Tokyo, Sect. 1a, Math. 31 (1984), 195-221.

[23] Tamura, Y., Free energy and the convergence of distributions of diffusion processes of McKean type, J. Fac. Sci. Univ. Tokyo, Sect. 1a, Math. 34 (1987), 443-484.

[24] Vintschger, R.V., The existence of invariant measures for $C[0,1]$-valued diffusions, Probab. Th. Rel. Fields 82 (1989), 307-313.

[25] Wonham, W.M., Lyapunov criteria for weak stochastic stability, J. Differential Equations 2 (1966), 195-207.

[26] Zakai, M., A Lyapunov criterion for the existence of stationary probability distributions for systems perturbed by noise, SIAM J. Control 7 (1969), 390-397.

[27] Zeidler, E., Nonlinear Functional Analysis and Its Applications I, Springer-Verlag 1986. 


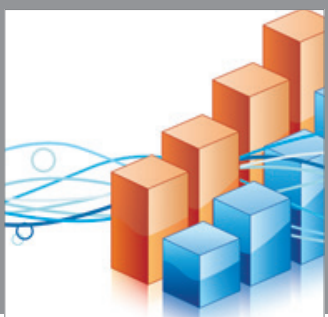

Advances in

Operations Research

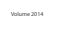

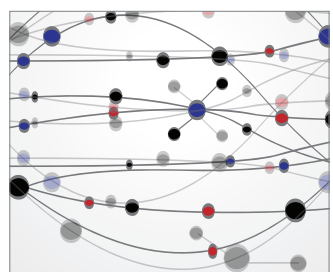

\section{The Scientific} World Journal
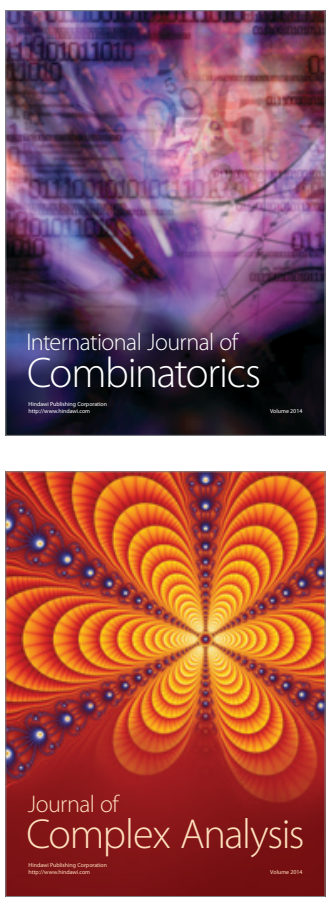

International Journal of

Mathematics and

Mathematical

Sciences
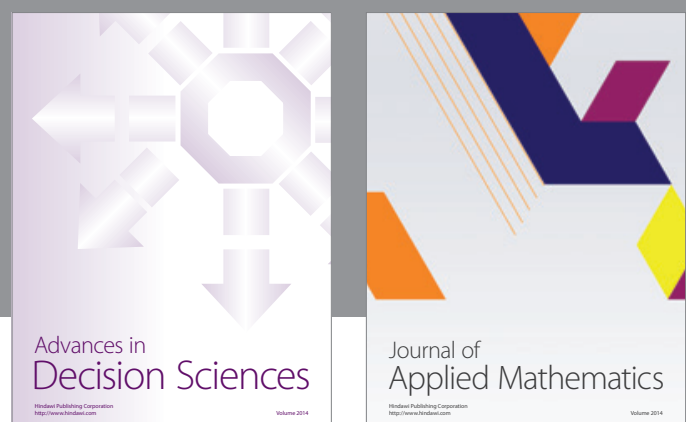

Journal of

Applied Mathematics
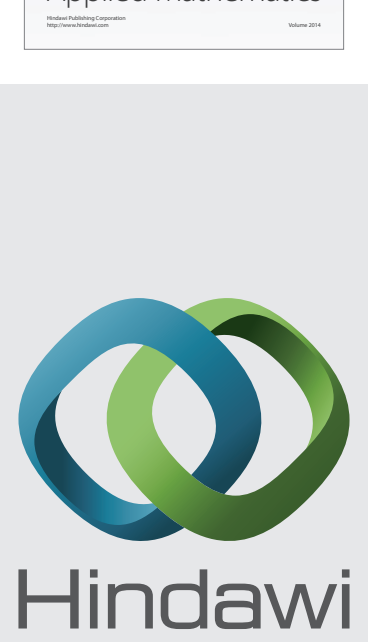

Submit your manuscripts at http://www.hindawi.com
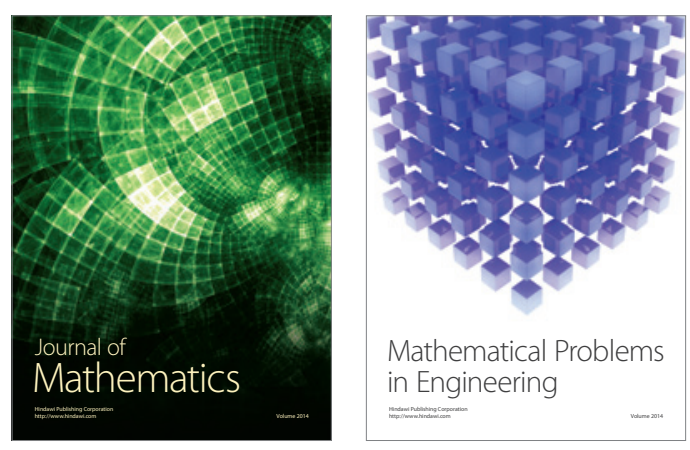

Mathematical Problems in Engineering
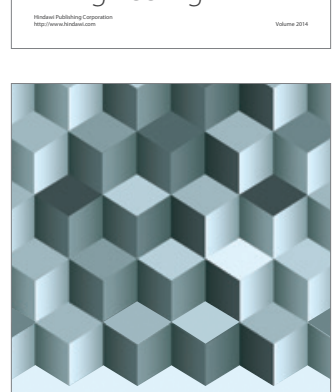

Journal of

Function Spaces
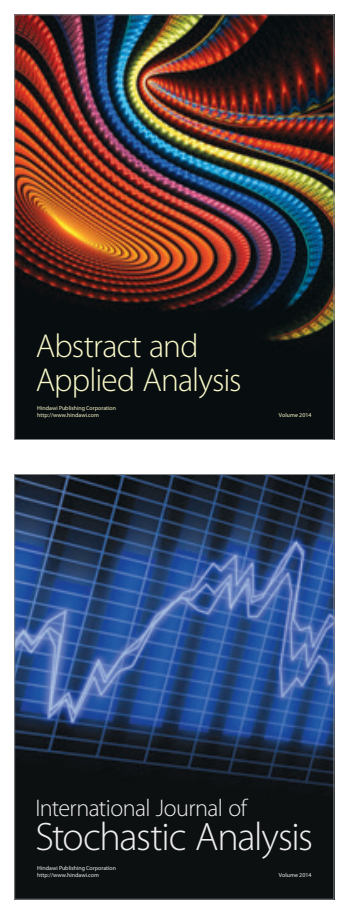

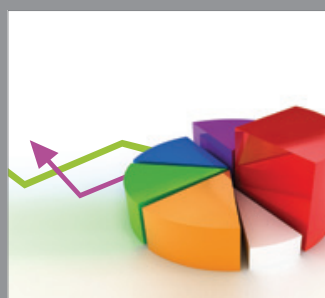

ournal of

Probability and Statistics

Promensencen
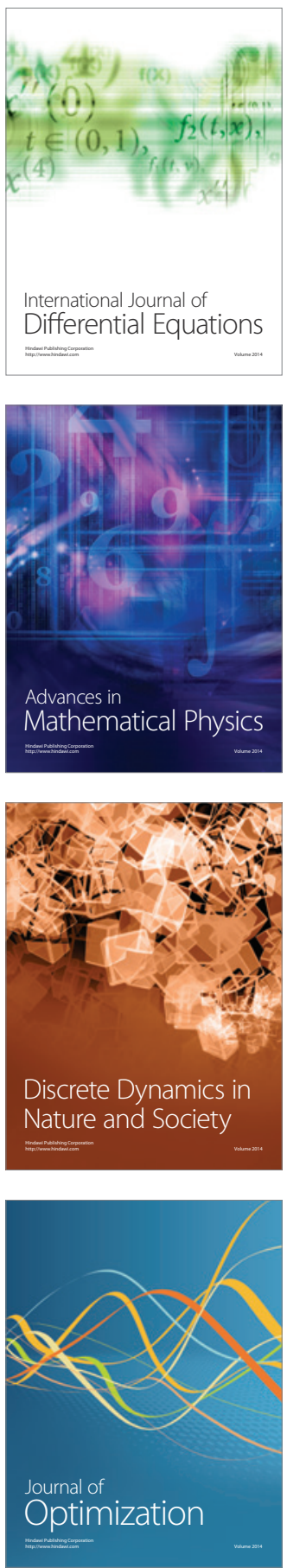